\section{Pattern Recognition Neuroradiology}

\author{
N.M. Borden and S.E. Forseen
}

New York, NY: Cambridge University Press, 2011, 339 pages, $\$ 84$

Despite advanced imaging techniques, a confident diagnosis also requires knowledge of the patient, the clinical data, and the lesion location. Authors of this user-friendly book are alumni of Barrow Neurologic Institute and provide the tools we need to arrive at the correct diagnosis or a reasonable differential diagnosis. This book includes basic information often omitted from other books: a practical method of image analysis, sample dictation templates, and a concise outline of didactic information regarding lesions or diseases. The image galleries show more than 700 high-quality representative CT and MR example images of the diseases discussed.

The book is divided into 2 major sections, the first being 6 chapters devoted to the brain and the second being 5 chapters on the spinal cord. Each section begins with a chapter dealing with basic concepts and terminology. The chapter on the brain summarizes how MR and CT images are produced and how the different signals interact with brain tissues. It also defines basic neurologic terms needed to interpret images. These are particularly helpful for trainees. The chapters that follow in both sections categorize neurologic diseases and their locations. An appendix provides dictation templates based on the systemic approach.

This brief, conversational book is rich in images that offer a comprehensive look at common and rare neurologic lesions. Therefore, neuroradiologists, neurologists, and neurosurgeons, regardless of their level of expertise, will not be disappointed. The book will be an invaluable diagnostic resource for medical students, trainees in neuroscience disciplines, radiologists, and individuals preparing for the qualifying examination on neuroradiology.

\section{E. Edmund Kim \\ University of California at Irvine \\ 101 The City Dr. S. \\ Orange, CA 92868 \\ E-mail: edmundek@uci.edu}

Published online Dec. 18, 2012.

DOI: 10.2967/jnumed.112.116202

\section{Nuclear Pharmacy Quick Reference}

C.N. Cole, S.M. Shaw, and R.J. Kowalsky

Washington, DC: American Pharmacists Association, 2012, 150 pages, $\$ 30.95$

Nuclear Pharmacy Quick Reference is a practical and informative text on radiopharmaceuticals. This book is authored by experienced and wellrespected individuals in the nuclear pharmacy community. The intent of the authors was to deliver a book that could be a source of readily accessible information, and that goal was realized. The book offers essential and pertinent information on radiopharmaceuticals, organized in

COPYRIGHT (C 2013 by the Society of Nuclear Medicine and Molecular Imaging, Inc. a concise fashion, and thus proves to be a practical, rapidly accessible reference source for the health-care provider.

The book is compact and lightweight, containing 8 chapters in a total of 150 pages. The information either is presented in well-organized tables or is summarized by bullet points. The text begins with a chapter that includes a reference table on radiopharmaceuticals, highlighting dosage forms, use, typical dose, and route of administration. A second table follows, providing an encompassing list of radionuclides and their nuclear properties. This table offers adequate information on the physical properties of radionuclides that may be used in nuclear imaging. The second chapter, "Radiopharmaceuticals and Procedures," compares different radiopharmaceuticals used in specific imaging procedures. For example, Table 2-3, "Biologic Properties of Renal Imaging Radiopharmaceuticals," lists 5 radiopharmaceuticals commonly used for renal imaging and their properties. From the table, it can easily be concluded that ${ }^{99 \mathrm{~m}} \mathrm{Tc}$-mercaptoacetyltriglycine undergoes renal excretion via tubular secretion, versus ${ }^{99 \mathrm{~m}} \mathrm{Tc}$-diethylenetriaminepentaacetic acid, which relies on glomerular filtration for excretion. Thus, a decision can quickly be made that ${ }^{99} \mathrm{~m}$ Tc-mercaptoacetyltriglycine is the better agent for visualizing the renal collecting system. It should be noted that although Table 2-4, "Comparison of ${ }^{67} \mathrm{Ga}$-Citrate vs. ${ }^{99 \mathrm{~m}} \mathrm{Tc}-\mathrm{vs}$. ${ }^{111}$ In-WBCs for Infection Labeling," is an invaluable tool, the same table appears in both Chapter 2 and Chapter 3.

Chapter 4, "Quality Control," follows the same summarizing-table format as the previous chapters. The authors successfully extract pertinent information from the package inserts of the radiopharmaceuticals and organize the information in a straightforward manner. With the addition of the "Special Instructions" column, the authors include comprehensive, vital information on different radiopharmaceuticals. The user can reference pearls of practice by locating the corresponding letter notations at the bottom of the table. The following chapter, "Radiation Dose Information," focuses on the average effective dose for different radiologic procedures. The table in this chapter provides a good reference to address radiation exposure or safety inquiries. Furthermore, this chapter also includes guidelines on special instructions or documentation needed for breast-feeding patients who are receiving radiopharmaceuticals.

The next chapter highlights information on the regulation of radiopharmaceuticals and on regulatory agencies significant to the practice of nuclear pharmacy. The remaining chapters cover drug interactions in nuclear medicine procedures and the clinical applications of radiopharmaceuticals. Although the authors recognize that the presented list of drug interactions is not comprehensive, the information serves as a great foundation to begin an investigation on the causes for altered biodistribution in a radiopharmaceutical. The chapter on clinical applications delivers a good overview of commonly used radiopharmaceuticals, organized by organ system. A review of the anatomy and physiology of each organ system is followed by essential information on the radiotracer and its clinical application.

Nuclear Pharmacy Quick Reference was intended to be a rapidly accessible reference of useful information, and its content and organization proves to have satisfied the authors' goal. It is a valuable tool for individuals working in the nuclear medicine department or the nuclear pharmacy.

\section{Dao Le}

M.D. Anderson Cancer Center

1515 Holcombe Blvd.

Houston, TX 77030

E-mail: dble@mdanderson.org

Published online Dec. 21, 2012.

DOI: 10.2967/jnumed.112.116194 